Supporting Information

\title{
Characteristics of Non-Fullerene Acceptor-Based Organic Photovoltaic Active Layers Using X-ray Scattering and Solid-state NMR
}

Yao Wu, ${ }^{\dagger}$ Qin Hu, ${ }^{\dagger}, \S$ Pu Fan, ${ }^{\dagger}$ Yufeng Jiang, ${ }^{\ddagger}$ Wenkai Zhong, $\|$ Weiguo Hu, ${ }^{*} \dagger$ Thomas P. Russell $*, \dagger$,

${ }^{\dagger}$ Department of Polymer Science and Engineering, University of Massachusetts Amherst, 120 Governors Drive, Amherst, Massachusetts 01003, United States

\$ Materials Sciences Division, Lawrence Berkeley National Laboratory, 1 Cyclotron Road, Berkeley, California 94720, United States

$\S$ School of Microelectronics, University of Science and Technology of China, Hefei, Anhui 230026, China

${ }^{\|}$Frontiers Science Center for Transformative Molecules, In-situ Center for Physical Science, and Center of Hydrogen Science, School of Chemistry and Chemical Engineering, Shanghai Jiao Tong University, Shanghai 200240, China

Keywords: X-ray scattering; solid-state nuclear magnetic resonance; relaxation measurement; non-fullerene acceptors; organic solar cells. 


\section{Experimental Section}

\section{Materials}

Unless stated otherwise, all the solvents and chemical reagents used were obtained commercially and were used without further purification. PTB7, PTB7-Th and ITIC were purchased from 1Material Inc.

\section{Fabrication and characterization of organic solar cells}

Photovoltaic devices were fabricated by spin-coating PEDOT:PSS (CLEVIOSTM P VP AI 4083) onto pre-cleaned glass substrates $(14.7 \times 14.7 \mathrm{~mm})$, patterned with ITO film $(10 \Omega / \square$, from Thin Film Devices, Inc.). The PEDOT:PSS-coated substrates were baked at $150{ }^{\circ} \mathrm{C}$ for 15 minutes in air, then transferred to an inert atmosphere glove box (N2 atmosphere, < 1 ppm O2, <1 ppm H2O) for deposition of the photoactive layer, interlayer, and top electrode. A mixture of PTB7 and ITIC (1:1 weight ratio) in chlorobenzene (CB), or a mixture of PTB7-Th and ITIC (1:1 weight ratio) in $\mathrm{CB}$, were stirred at room temperature for $\sim 12$ hours. The photoactive layers were deposited by spin-coating the solution onto the PEDOT:PSS layer. The thickness of the active layer film was $\sim 100 \mathrm{~nm}$ as determined by profilometry. The interlayers spin-coated on the active layer from solutions $(4 \mathrm{mg} / \mathrm{mL})$ at constant spin-coating speed of $4000 \mathrm{rpm}$. Thermal evaporation of $\mathrm{Ag}$ electrodes was performed through a shadow mask, defining a maximum available electrode area of $0.06 \mathrm{~cm}^{2}$.

\section{Characterization}

\section{UV-vis absorption spectra}

UV-vis absorption spectra were measured using a Shimadzu UV-3600 spectrophotometer. Samples were spin-coated on the glass/PEDOT:PSS substrates, using the same condition as device 
fabrication. The absorption of PEDOT:PSS was subtracted to isolate the absorption from the samples.

\section{Ultraviolet photoelectron spectroscopy}

UPS samples were spin-coated on the glass/ITO/PEDOT:PSS substrates, using the same condition as device fabrication. UPS measurements were performed on the Omicron Nanotechnology Model $\mathrm{ESCA}+\mathrm{S}$, consisting of a helium discharge lamp (He I line, $21.2 \mathrm{eV}$ ) as the UV excitation source and a hemispherical SPHERA energy analyzer. All samples were biased by $-3 \mathrm{~V}$ to compensate for the instrument work function difference repelling the low kinetic energy electrons, shifting the energy scale of experimental graphs by $3 \mathrm{eV}$. The detail calculation of the HOMO levels was used the same methods as ref. 1 . The first step is to determine the position of the secondary electron cut-off (Esec) energy. Then, the position of a vacuum level is located $21.2 \mathrm{eV}$ away from the $\mathrm{E}_{\text {sec. }}$ The HOMO energy would be the difference between the vacuum level and the HOMO onset.

\section{Grazing Incidence wide-angle X-ray Scattering}

Grazing incidence x-ray diffraction (GIXD) characterization of the active layer was performed at beamline 7.3.3, Advanced Light Source (ALS), Lawrence Berkeley National Lab (LBNL). X-ray energy was $10 \mathrm{keV}$ and operated in top-off mode. The scattering intensity was recorded on a $2 \mathrm{D}$ image plate (Pilatus 2M) with a pixel size of $0.172 \mathrm{~mm}$ by $0.172 \mathrm{~mm}$. The samples were $\sim 10 \mathrm{~mm}$ long in the direction of the beam path, and the detector was located at a distance of $270 \mathrm{~mm}$ from the sample center (distance calibrated by $\mathrm{AgB}$ reference). The incidence angle was chosen to be $0.16^{\circ}$ (above critical angle) for GIXD measurement. ${ }^{2-4}$ OSC samples were prepared on PEDOT:PSS similarly covered Si wafers to the OSC devices. The coherence length (Lc) of all the

films was calculated from $1 \mathrm{D}$ profiles by Scherrer equation ${ }^{5}: \mathrm{Lc}=2 \pi \mathrm{K} / \Delta \mathrm{q}$, where $\Delta \mathrm{q}$ is the fullwidth at half-maximum of the peak and $\mathrm{K}$ is a shape factor ( 0.9 was used here). 
All the strong peaks were fitted by Gaussion methods, which are shown in Fig S3. Other peak positions were simply determined by maximum intensity in that region due to the peak broadening, weak intensity or asymmetric peak shapes.

\section{Resonant Soft X-ray Scattering}

RSoXS was performed at beamline 11.0.1.2 ALS, LBNL. Samples for R-SoXS measurements were prepared on a PEDOT: PSS modified Si substrate under the same conditions as used for OSC device fabrication, and then transferred by floating in water to a $1.5 \mathrm{~mm} \times 1.5 \mathrm{~mm}, 100$-nm thick $\mathrm{Si}_{3} \mathrm{~N}_{4}$ membrane supported by a $5 \mathrm{~mm} \times 5 \mathrm{~mm}, 200 \mathrm{~mm}$ thick Si frame (Norcada Inc.).

\section{Solid-State NMR}

Sample preparation

As-received samples are the original materials obtained from the manufacture without any further processing. All the other as-cast samples were made by the following steps. Materials were dissolved in the solvent and stirred at room temperature. The films were cast onto glass substrate and transferred to the surface of water. Then the films were collected, cut to small pieces, and placed in the NMR rotors.

${ }^{13} \mathrm{C}$ Solid-State NMR measurement:

All solid-state NMR experiments were conducted on a Bruker $600 \mathrm{MHz}$ AvanceIII spectrometer, operating at a ${ }^{1} \mathrm{H}$ frequency of $600.13 \mathrm{MHz}$ and ${ }^{13} \mathrm{C}$ frequency of $150.90 \mathrm{MHz}$, in $4 \mathrm{~mm} \mathrm{CP} / \mathrm{MAS}$ probes. Several techniques were employed: (1) CP/MAS with optional $\mathrm{T}_{1 \rho}$ measurement. The pulse sequence was a $90^{\circ}$ pulse followed by an optional ${ }^{1} \mathrm{H}$ spin lock pulse, then a cross polarization (CP) period, then ${ }^{13} \mathrm{C}$ detection with high-power ${ }^{1} \mathrm{H}$ decoupling. (2) ${ }^{1} \mathrm{H} \mathrm{T}_{1}$ measurement with ${ }^{13} \mathrm{C}$ detection. The pulse sequence was a ${ }^{1} \mathrm{H}$ saturation pulse train followed by a variable recovery delay, then ${ }^{13} \mathrm{C}$ detection by cross polarization. The chemical shift was externally referenced by the non-protonated aromatic peak of para-di-tert-butylbenzene at 148.8 ppm. A RAMP-CP (ramped-amplitude cross-polarization) scheme was used for cross-polarization. 
The contact time was between 0.2 and $3 \mathrm{~ms}$. The recycle delay, decoupling field, and spinning speed were $3 \mathrm{~s}, 78 \mathrm{kHz}$, and $8-9.5 \mathrm{kHz}$, respectively.
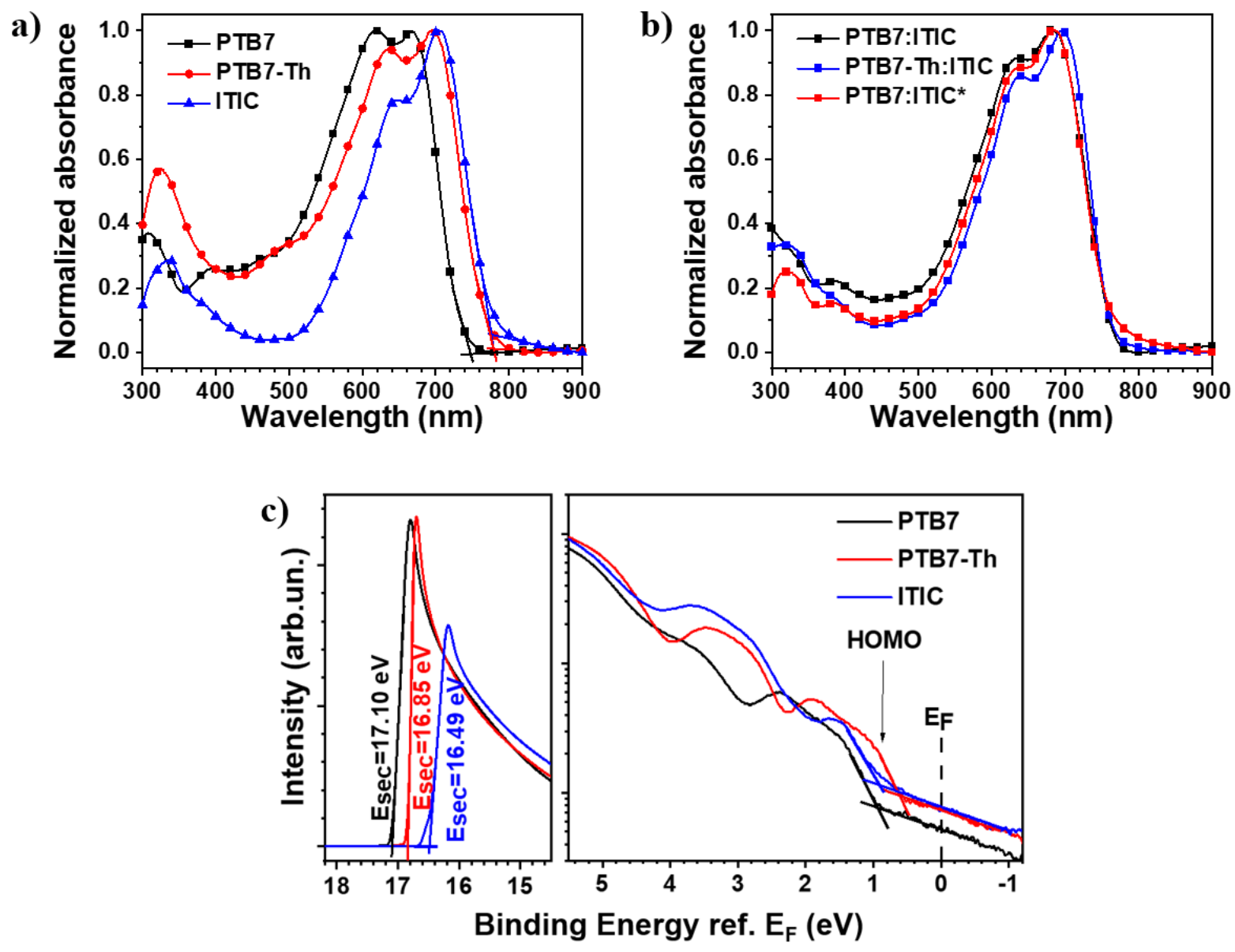

Figure S1. Absorption spectra of (a) pure and (b) blend films. (c) UPS spectra of PTB7, PTB7-Th and ITIC. Left and right panes of each part of the figure show the energy range near the secondary electron cutoff and Fermi/HOMO energy level, respectively. 

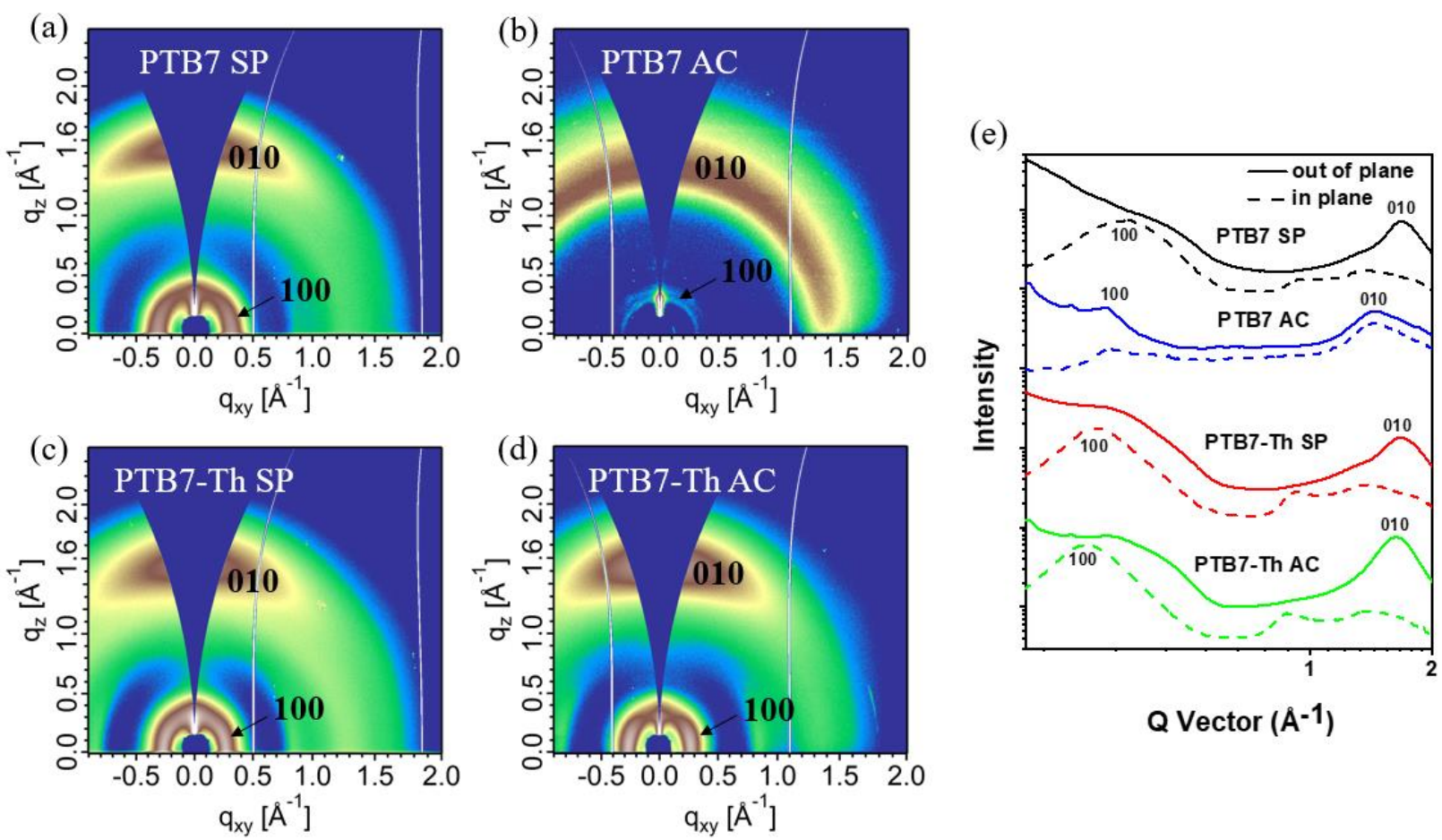

Figure S2. 2D GIWAXS patterns of a) PTB7 SP, b) PTB7 AC, c) PTB7-Th SP, and d) PTB7-Th AC; e) Scattering profiles of out-of-plane and in-plane for PTB7 and PTB7-Th. SP:spin-coating; AC: as-cast.
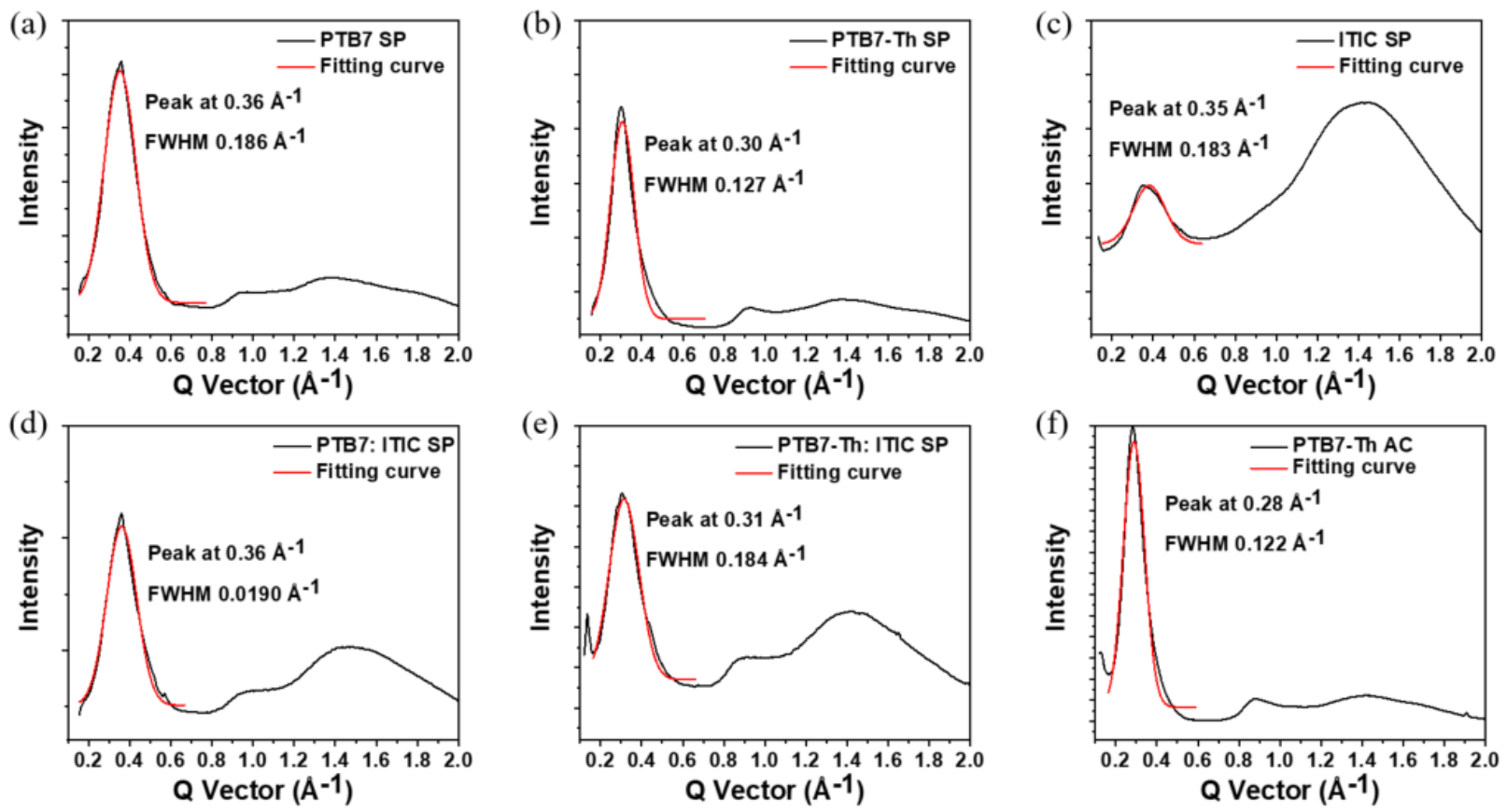
Figure S3. Scattering profiles of a) PTB7 SP, b) PTB7-Th SP, c)ITIC SP, d) PTB7:ITIC SP, e) PTB7-Th:ITIC SP, and f) PTB7-Th AC with fitting curves of (100) peaks at the in plane directions. SP:spin-coating; AC: as-cast.
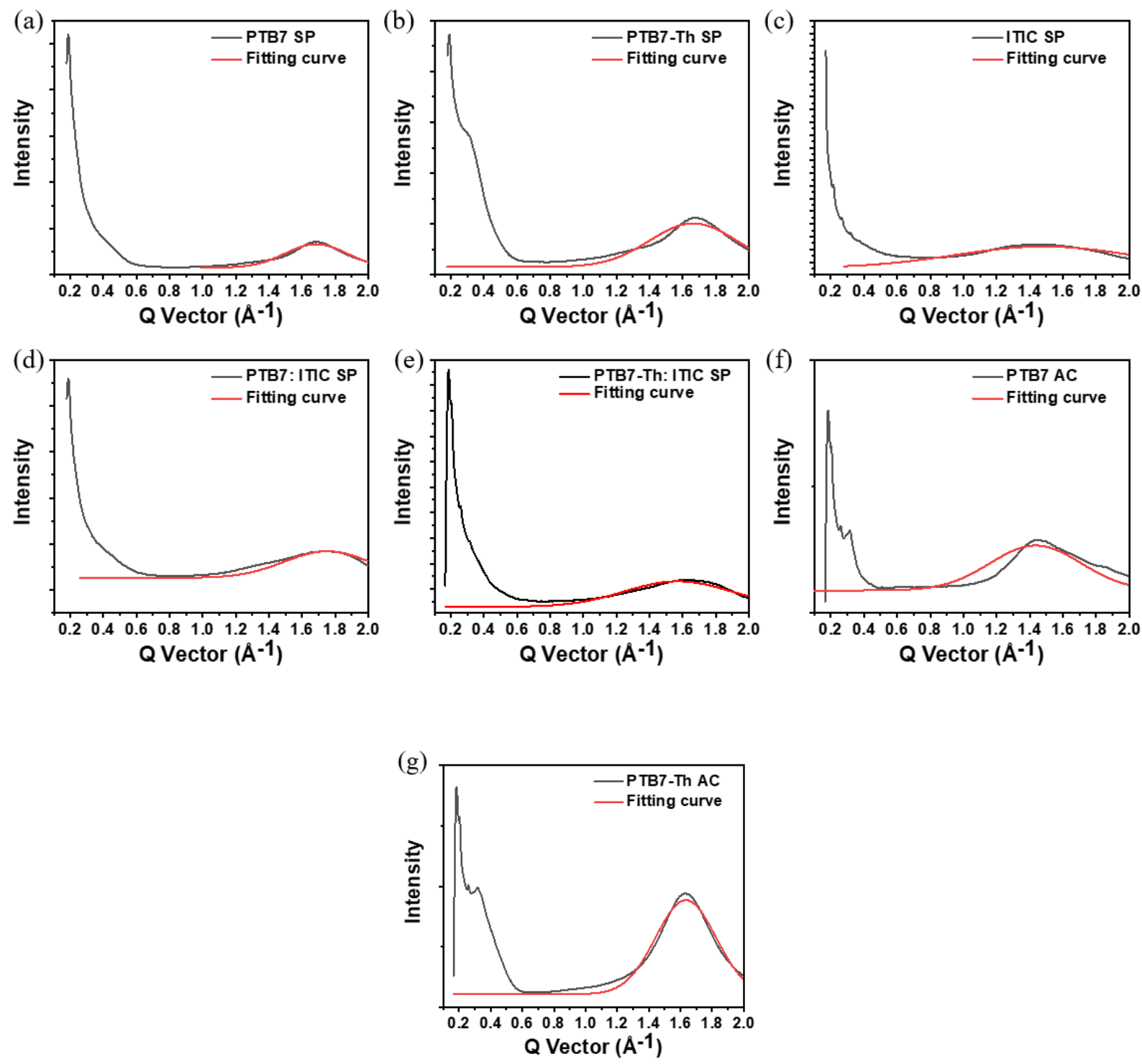

Figure S4. Scattering profiles of a) PTB7 SP, b) PTB7-Th SP, c)ITIC SP, d) PTB7:ITIC SP, e) PTB7-Th:ITIC SP, f) PTB7 AC and g) PTB7-Th AC with fitting curves of (010) peaks at the out of plane directions. SP:spin-coating; AC: as-cast. 

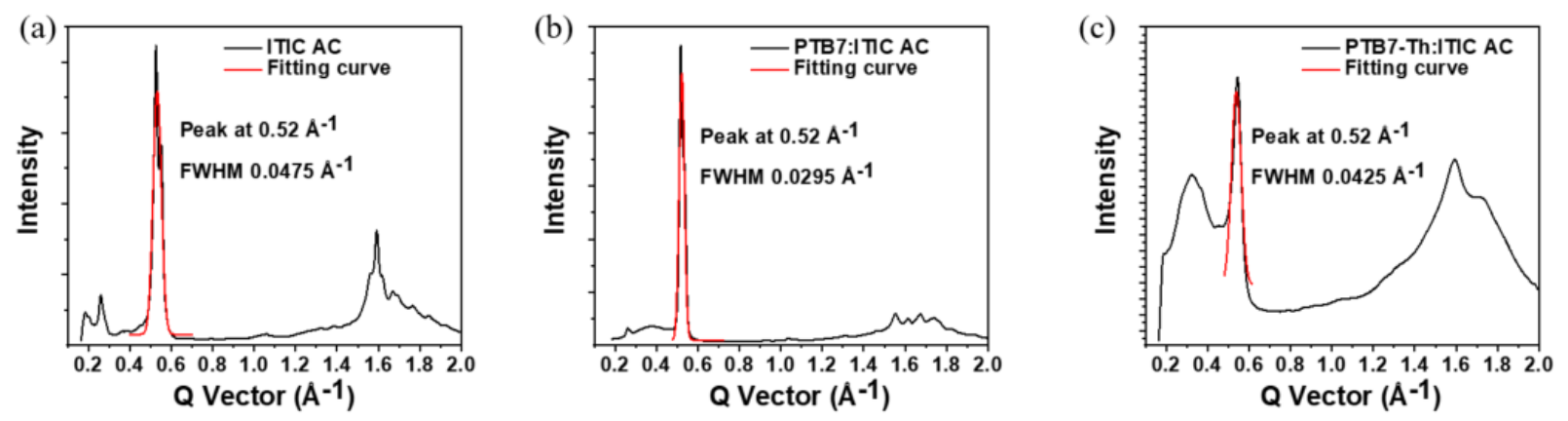

Figure S5. Scattering profiles of a) ITIC AC, b) PTB7:ITIC AC and c) PTB7-Th:ITIC AC with fitting curves of the peaks at $0.52 \AA^{-1}$ at the out of plane directions. SP:spin-coating; AC: as-cast.

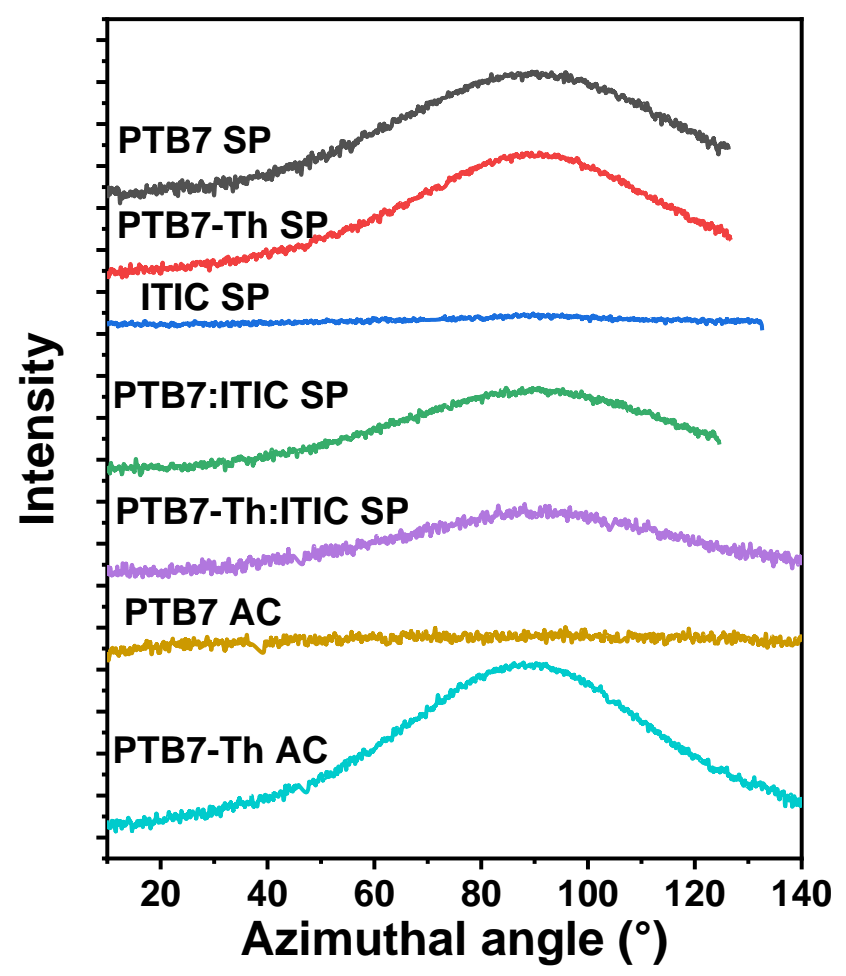

Figure S6. Azimuthal angle distribution of (010) peaks of PTB7 SP, PTB7-Th SP, ITIC SP, PTB7:ITIC SP, PTB7-Th:ITIC SP, PTB7 AC and PTB7-Th AC. Only ITIC SP and PTB7 AC do not show preferred orientation. SP:spin-coating; AC: as-cast. 

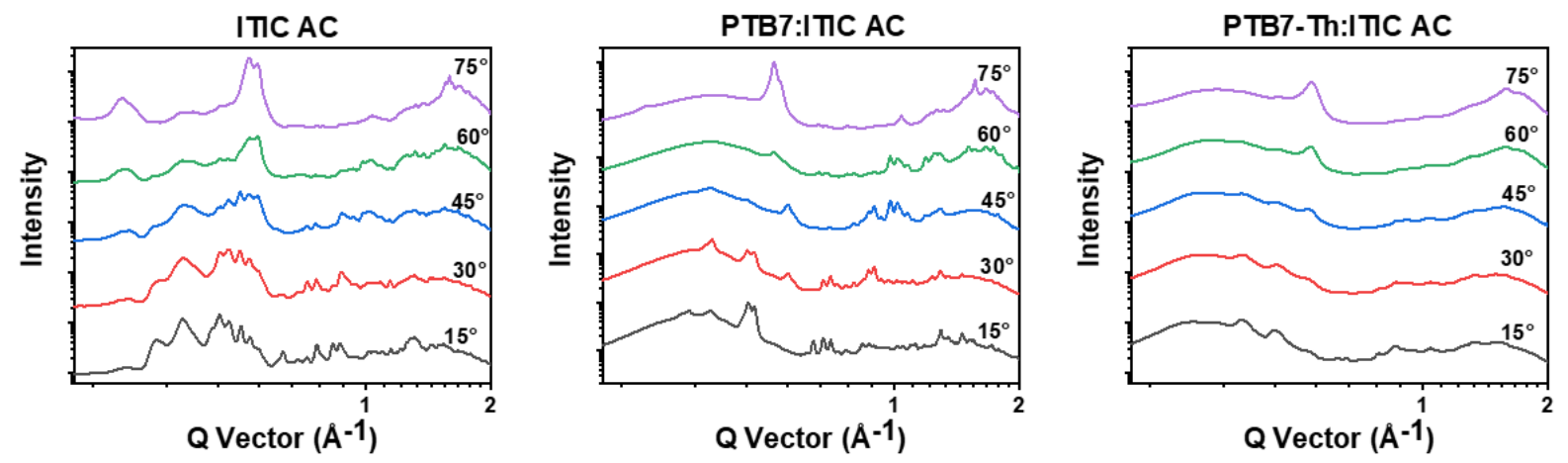

Figure S7. Scattering profiles of ITIC AC, PTB7:ITIC AC and PTB7-Th:ITIC AC in different azimuthal angles. The width of the sectors are $10^{\circ}$. SP:spin-coating; AC: as-cast.
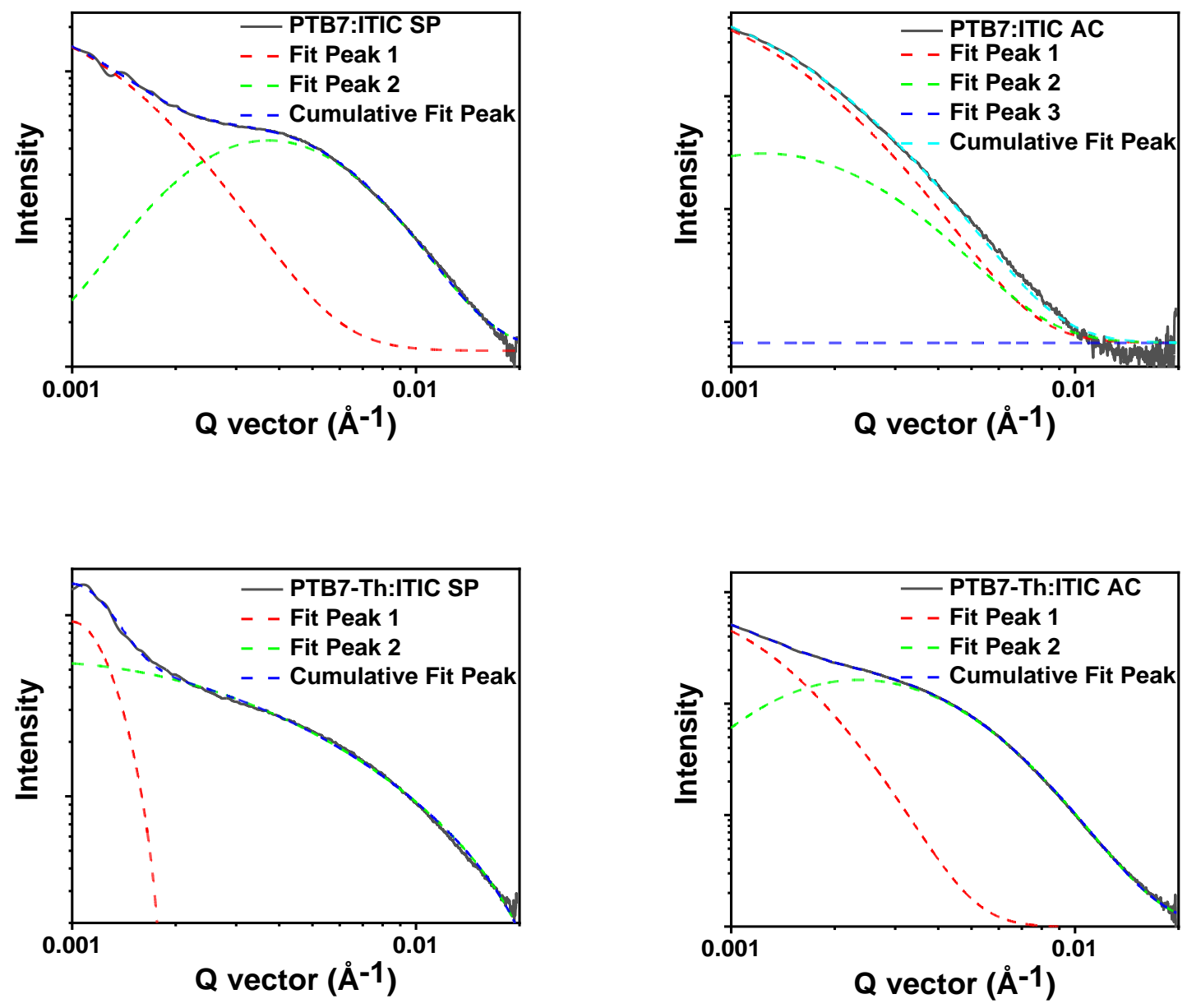
Figure S8. The fitted R-SoXS scattering profiles with log-normal functions. ${ }^{6}$ SP: Spin-coating; AC: as-cast.
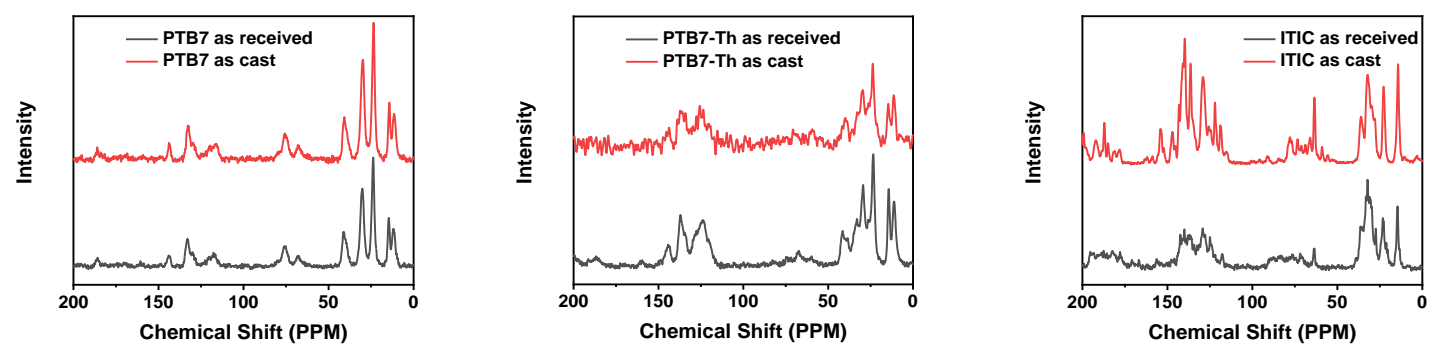

Figure S9. CP/MAS ${ }^{13} \mathrm{C}$ ssNMR spectrum of PTB7, PTB7-Th, and ITIC as received (black) and as cast (red) samples.

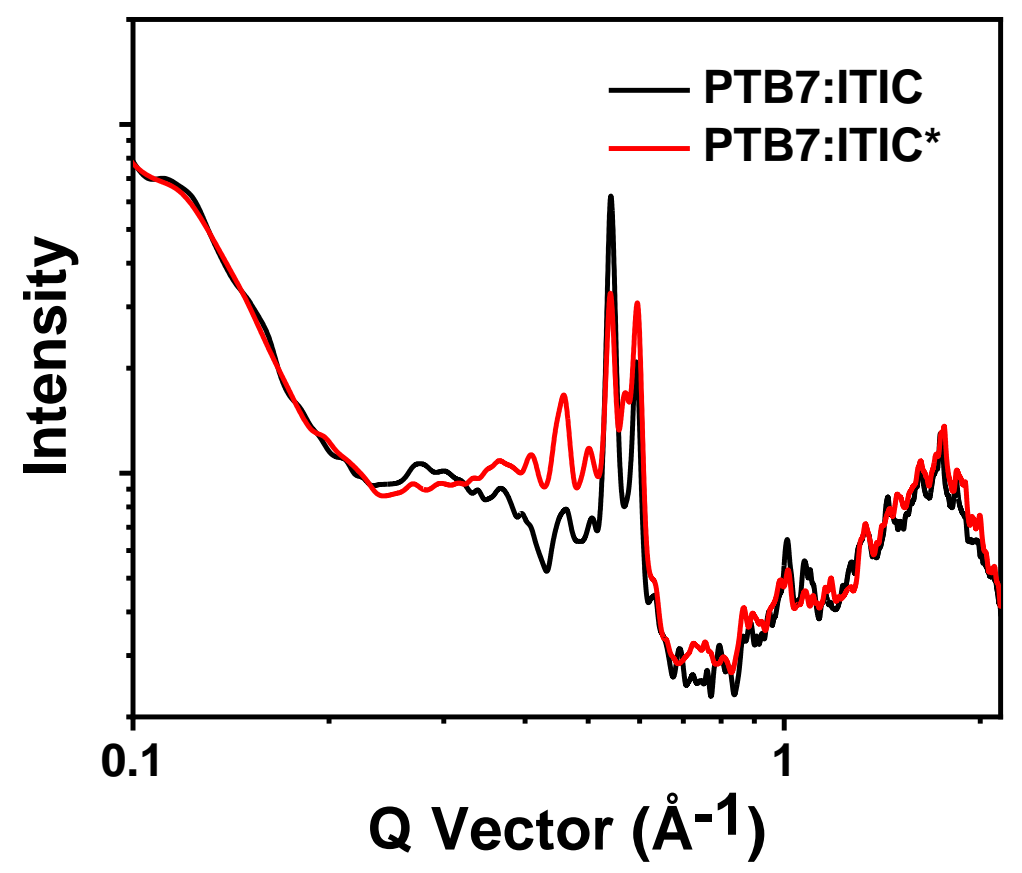

Figure S10. Wide-angle X-ray scattering profiles of PTB7:ITIC and PTB7:ITIC*. The curves were extracted from a circular average signals from all the diretions. 


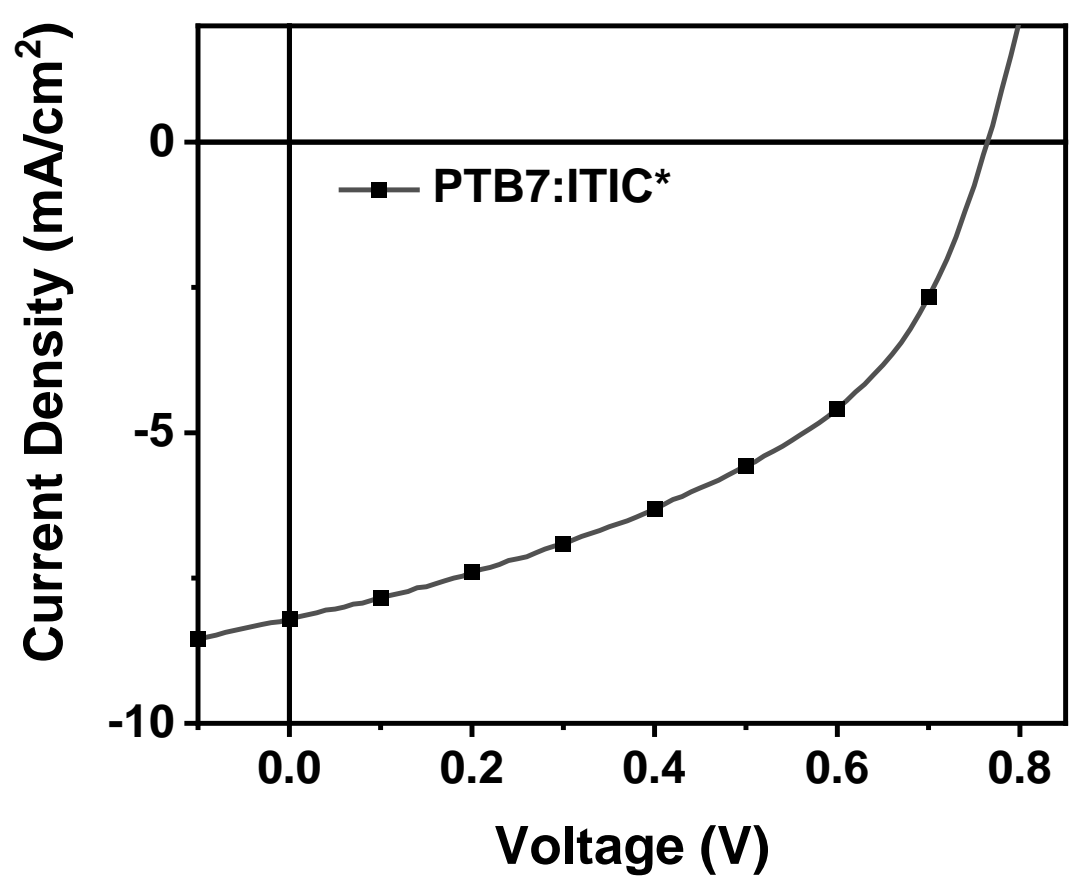

Figure S11. $J-V$ curves of optimized devices based on PTB7:ITIC*.

Table S1. Optical and electronic properties of PTB7, PTB7-Th and ITIC.

\begin{tabular}{c|cccccc}
\hline Materials & $\begin{array}{c}\lambda_{\max } \\
(\mathrm{nm})\end{array}$ & $\begin{array}{c}E_{\mathrm{g}}{ }_{\mathrm{g} t} \\
(\mathrm{eV})\end{array}$ & $\begin{array}{c}\mathrm{E}_{\text {sec }} \\
(\mathrm{eV})\end{array}$ & $\begin{array}{c}\Phi_{\text {hole }} \\
(\mathrm{eV})\end{array}$ & $\begin{array}{c}\text { HOMO } \\
(\mathrm{eV})\end{array}$ & $\begin{array}{c}\text { LUMO } \\
(\mathrm{eV})\end{array}$ \\
\hline PTB7 & 667 & 1.66 & 17.10 & 0.94 & -5.04 & -3.38 \\
\hline PTB7-Th & 695 & 1.59 & 16.85 & 0.61 & -4.96 & -3.37 \\
\hline ITIC & 704 & 1.60 & 16.49 & 0.92 & -5.63 & -4.03 \\
\hline
\end{tabular}

Table S2. $T_{1 \rho}(\mathrm{ms})$ of several peaks of PTB7 as a function of spinning speed. All the decays were fitted with single-exponential models. The 1-sigma fitting error for all the values are $\pm 0.1-0.2 \mathrm{~ms}$.

\begin{tabular}{|c|c|c|c|}
\hline $\begin{array}{c}\text { peak position } \\
(\mathrm{ppm})\end{array}$ & $5 \mathrm{kHz}$ & $7.5 \mathrm{kHz}$ & $9.5 \mathrm{kHz}$ \\
\hline 12 & 3.0 & 3.3 & 3.7 \\
\hline 15 & 3.4 & 3.9 & 4.0 \\
\hline 18 & 2.9 & 3.1 & 3.3 \\
\hline
\end{tabular}




\begin{tabular}{|c|c|c|c|}
\hline 24 & 2.9 & 3.0 & 3.2 \\
\hline 35 & 2.8 & 2.8 & 3.0 \\
\hline 70 & 2.8 & 3.0 & 3.1 \\
\hline 127 & 3.0 & 3.2 & 3.8 \\
\hline
\end{tabular}

Table S3. $\mathrm{T}_{1 \rho}$ relaxation times (ms) of PTB7, PTB7-Th, and ITIC in the neat and blend forms. All the decays were fitted with single-exponential models. The error bars are 1-sigma fitting errors.

\begin{tabular}{|c|c|c|c|c|c|c|c|c|}
\hline & \multicolumn{5}{|c|}{ Donor $^{\mathrm{c}}$} & \multicolumn{3}{|c|}{ Acceptor $^{\mathrm{d}}$} \\
\hline Samples/ms & $11 \mathrm{ppm}$ & $24 \mathrm{ppm}$ & $41 \mathrm{ppm}$ & $120 \mathrm{ppm}$ & $133 \mathrm{ppm}$ & $23 \mathrm{ppm}$ & $36 \mathrm{ppm}$ & $140 \mathrm{ppm}$ \\
\hline $\mathrm{PTB}^{\mathrm{a}}$ & $3.5 \pm 0.2$ & $2.8 \pm 0.1$ & $2.5 \pm 0.1$ & & $3.5 \pm 0.2$ & & & \\
\hline $\mathrm{PTB}^{\mathrm{b}}$ & $2.6 \pm 0.1$ & & & & & & & \\
\hline PTB7-Th ${ }^{\mathrm{a}}$ & & $1.3 \pm 0.1$ & & & $2.3 \pm 0.2$ & & & \\
\hline PTB7-Th ${ }^{\mathrm{b}}$ & $2.4 \pm 0.1$ & $1.9 \pm 0.1$ & & $3.0 \pm 0.1$ & & & & \\
\hline PTB7:ITIC ${ }^{a}$ & $3.9 \pm 0.1$ & & $2.9 \pm 0.1$ & & & & $11.2 \pm 0.8$ & $15.3 \pm 0.3$ \\
\hline PTB7:ITIC*a & $4.9 \pm 0.5$ & & $3.0 \pm 0.2$ & & & & $5.7 \pm 0.6$ & $7.8 \pm 0.4$ \\
\hline PTB7-Th:ITIC ${ }^{a}$ & $3.3 \pm 0.2$ & & $1.4 \pm 0.2$ & & & & $10.9 \pm 0.9$ & $13.4 \pm 0.2$ \\
\hline ITIC $^{\mathrm{a}}$ & & & & & & $9.5 \pm 0.6$ & $9.3 \pm 0.4$ & $10.8 \pm 0.3$ \\
\hline ITIC $^{\mathrm{b}}$ & & & & & & $15 \pm 1$ & & \\
\hline
\end{tabular}

${ }^{\mathrm{a}}$ as-cast samples; ${ }^{\mathrm{b}}$ as-received samples; ${ }^{\mathrm{c}}$ The signals are from PTB7 or PTB7-Th; ${ }^{\mathrm{d}}$ The signals are from ITIC. PTB7:ITIC* was processed from mixed solvent (chlorobenzene:benzene=1:1); Other as-cast samples were processed from pure chlorobenzene.

Table S4. $\mathrm{T}_{1}$ relaxation times (s) of PTB7, PTB7-Th, and ITIC in the neat and blend forms. All the decays were fitted with single-exponential models. The error bars are 1-sigma fitting errors.

\begin{tabular}{|c|c|c|c|c|c|c|c|c|c|c|}
\hline \multirow{2}{*}{ Samples/ms } & \multicolumn{6}{|c|}{ Donor $^{\mathrm{c}}$} & \multicolumn{4}{|c|}{ Acceptor $^{d}$} \\
\hline & $11 \mathrm{ppm}$ & $24 \mathrm{ppm}$ & 30ppm & $41 \mathrm{ppm}$ & 133 ppm & $137 \mathrm{ppm}$ & $23 \mathrm{ppm}$ & $36 \mathrm{ppm}$ & $137 \mathrm{ppm}$ & $140 \mathrm{ppm}$ \\
\hline ITIC $^{a}$ & & & & & & & \multicolumn{3}{|c|}{$0.97 \pm 0.041 .06 \pm 0.04$} & $1.03 \pm 0.03$ \\
\hline $\mathrm{PTB}^{\mathrm{a}}$ & \multicolumn{3}{|l|}{$0.93 \pm 0.04$} & \multicolumn{3}{|c|}{$1.04 \pm 0.051 .05 \pm 0.04$} & & & & \\
\hline PTB $^{\mathrm{a}}$ & \multicolumn{6}{|c|}{$0.93 \pm 0.010 .96 \pm 0.020 .91 \pm 0.03$} & & & & \\
\hline
\end{tabular}




\begin{tabular}{c|lc|cc}
\hline PTB7-Th $^{\mathrm{b}}$ & $1.13 \pm 0.061 .08 \pm 0.03$ & $1.17 \pm 0.04$ & & \\
\hline PTB7:ITIC & $0.90 \pm 0.04$ & $0.98 \pm 0.04$ & $0.66 \pm 0.04$ & $0.63 \pm 0.03$ \\
\hline PTB7:ITIC $^{* \mathrm{a}}$ & $1.16 \pm 0.08$ & $1.12 \pm 0.09$ & $1.28 \pm 0.091 .35 \pm 0.03$ \\
\hline PTB7-Th:ITIC & $1.04 \pm 0.02$ & $1.2 \pm 0.2$ & $0.80 \pm 0.03$ & $0.72 \pm 0.02$ \\
\hline
\end{tabular}

${ }^{\mathrm{a}}$ as cast samples; ${ }^{\mathrm{b}}$ as received samples; ${ }^{\mathrm{c}}$ The signals are from PTB7 or PTB7-Th; ${ }^{\mathrm{d}}$ The signals are from ITIC. PTB7:ITIC* was processed from mixed solvent (chlorobenzene:benzene=1:1); Other as-cast samples were processed from pure chlorobenzene.

Table S5. Performance of the Optimized OSC Devices Based on PTB7:ITIC*.

\begin{tabular}{ccccc}
\hline Active layer & $V_{\mathrm{OC}^{\mathrm{a}}}$ & $J_{\mathrm{SC}^{\mathrm{a}}}$ & $\mathrm{FF}^{\mathrm{a}}$ & $\mathrm{PCE}^{\mathrm{a}}$ \\
& $(\mathrm{V})$ & $(\mathrm{mA} \mathrm{cm})^{-2}$ & $(\%)$ & $(\%)$ \\
\hline PTB7:ITIC* $^{*}$ & $\begin{array}{c}0.760 \pm 0.012 \\
(0.772)\end{array}$ & $\begin{array}{c}8.00 \pm 0.13 \\
(8.19)\end{array}$ & $\begin{array}{c}44.1 \pm 1.2 \\
(45.2)\end{array}$ & $\begin{array}{c}2.68 \pm 0.14 \\
(2.82)\end{array}$
\end{tabular}

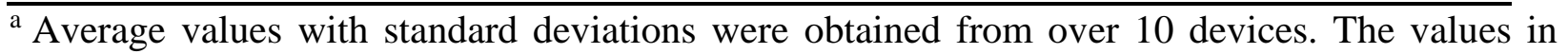
parentheses are the parameters from the best device.

(1) Page, Z. A.; Duzhko, V. V.; Emrick, T., Conjugated Thiophene-Containing Polymer Zwitterions: Direct Synthesis and Thin Film Electronic Properties. Macromolecules 2012, 46, 344351.

(2) Ilavsky, J.; Jemian, P. R., Irena: tool suite for modeling and analysis of small-angle scattering. J. Appl. Crystallogr. 2009, 42, 347-353.

(3) Nelson, A., Co-refinement of multiple-contrast neutron/X-ray reflectivity data using MOTOFIT. J. Appl. Crystallogr. 2006, 39, 273-276.

(4) Beaucage, G., Approximations Leading to a Unified Exponential/Power-Law Approach to Small-Angle Scattering. J. Appl. Crystallogr. 1995, 28, 717-728. 
(5) Rivnay, J.; Noriega, R.; Kline, R. J.; Salleo, A.; Toney, M. F., Quantitative analysis of lattice disorder and crystallite size in organic semiconductor thin films. Phys. Rev. B 2011, 84, 045203.

(6) Ma, W.; Tumbleston, J. R.; Ye, L.; Wang, C.; Hou, J.; Ade, H., Quantification of nanoand mesoscale phase separation and relation to donor and acceptor quantum efficiency, $\mathrm{J}(\mathrm{sc})$, and FF in polymer:fullerene solar cells. Adv. Mater. 2014, 26, 4234-41. 